\title{
Subgroup and Resistance Analyses of Raltegravir for Resistant HIV-1 Infection
}

\author{
David A. Cooper, M.D., D.Sc., Roy T. Steigbigel, M.D., Jose M. Gatell, M.D., Ph.D., \\ Jurgen K. Rockstroh, M.D., Christine Katlama, M.D., Patrick Yeni, M.D., \\ Adriano Lazzarin, M.D., Bonaventura Clotet, M.D., Princy N. Kumar, M.D., \\ Joseph E. Eron, M.D., Mauro Schechter, M.D., Ph.D., Martin Markowitz, M.D., \\ Mona R. Loutfy, M.D., M.P.H., Jeffrey L. Lennox, M.D., Jing Zhao, Ph.D., \\ Joshua Chen, Ph.D., Desmond M. Ryan, B.S., Rand R. Rhodes, M.S., \\ John A. Killar, M.S., Lucinda R. Gilde, B.S., Kim M. Strohmaier, B.S., \\ Anne R. Meibohm, Ph.D., Michael D. Miller, Ph.D., Daria J. Hazuda, Ph.D., \\ Michael L. Nessly, M.S., Mark J. DiNubile, M.D., Robin D. Isaacs, M.D., \\ Hedy Teppler, M.D., and Bach-Yen Nguyen, M.D., for the BENCHMRK Study Teams*
}

\section{A BSTRACT}

\section{BACKGROUND}

We evaluated the efficacy of raltegravir and the development of viral resistance in two identical trials involving patients who were infected with human immunodeficiency virus type 1 (HIV-1) with triple-class drug resistance and in whom antiretroviral therapy had failed.

\section{METHODS}

We conducted subgroup analyses of the data from week 48 in both studies according to baseline prognostic factors. Genotyping of the integrase gene was performed in raltegravir recipients who had virologic failure.

\section{RESULTS}

Virologic responses to raltegravir were consistently superior to responses to placebo, regardless of the baseline values of HIV-1 RNA level; CD4 cell count; genotypic or phenotypic sensitivity score; use or nonuse of darunavir, enfuvirtide, or both in optimized background therapy; or demographic characteristics. Among patients in the two studies combined who were using both enfuvirtide and darunavir for the first time, HIV-1 RNA levels of less than 50 copies per milliliter were achieved in $89 \%$ of raltegravir recipients and $68 \%$ of placebo recipients. HIV-1 RNA levels of less than 50 copies per milliliter were achieved in $69 \%$ and $80 \%$ of the raltegravir recipients and in $47 \%$ and $57 \%$ of the placebo recipients using either darunavir or enfuvirtide for the first time, respectively. At 48 weeks, 105 of the 462 raltegravir recipients (23\%) had virologic failure. Genotyping was performed in 94 raltegravir recipients with virologic failure. Integrase mutations known to be associated with phenotypic resistance to raltegravir arose during treatment in 64 patients (68\%). Forty-eight of these 64 patients $(75 \%)$ had two or more resistance-associated mutations.

\section{CONCLUSIONS}

When combined with an optimized background regimen in both studies, a consistently favorable treatment effect of raltegravir over placebo was shown in clinically relevant subgroups of patients, including those with baseline characteristics that typically predict a poor response to antiretroviral therapy: a high HIV-1 RNA level, low CD4 cell count, and low genotypic or phenotypic sensitivity score. (ClinicalTrials.gov numbers, NCT00293267 and NCT00293254.)
From the National Centre in HIV Epidemiology and Clinical Research, University of New South Wales, Sydney (D.A.C.); State University of New York at Stony Brook, Stony Brook (R.T.S.); University of Barcelona, Barcelona (J.M.G.); University of Bonn, Bonn, Germany (J.K.R.); Hospital Pitié-Salpêtrière, Université Pierre et Marie Curie, Paris (C.K.); Hospital BichatClaude Bernard, Paris (P.Y.); San Raffaele Scientific Institute, Milan (A.L.); Hospital Germans Trias i Pujol, Fundación Irsicaixa, Barcelona (B.C.); Georgetown University Medical Center, Washington, DC (P.N.K.); University of North Carolina, Chapel Hill (J.E.E.); Universidade Federal do Rio de Janeiro, Rio de Janeiro (M.S.); Aaron Diamond Research Center, Rockefeller University, New York (M.M.); University of Toronto, Toronto (M.R.L.); Emory University School of Medicine, Atlanta (J.L.L.); and Merck Research Laboratories, North Wales, PA (J.Z., J.C., D.M.R., R.R.R., J.A.K., L.R.G., K.M.S., A.R.M., M.D.M., D.J.H., M.L.N., M.J.D., R.D.I., H.T., B.-Y.N.). Address reprint requests to Dr. Nguyen at Merck Research Laboratories, P.O. Box 1000, UG3D-56, North Wales, PA 19454-1099, or at bachyen_nguyen@ merck.com.

\footnotetext{
*The members of the BENCHMRK (Blocking Integrase in Treatment Experienced Patients with a Novel Compound against HIV, Merck) study teams are listed in Supplementary Appendix 1, available with the full text of this article at www. nejm.org.
}

N Engl J Med 2008;359:355-65. Copyright $(2008$ Massachusetts Medical Society. 
ESPITE THE SUBSTANTIAL DECREASE IN mortality and morbidity rates associated with highly active antiretroviral therapy over the past decade, there is still a substantial need for effective antiretroviral drugs for patients infected with resistant human immunodeficiency virus type 1 (HIV-1). ${ }^{1,2}$ The majority of licensed antiretroviral drugs belong to three classes targeting either the HIV-1 protease or reverse transcriptase, and considerable cross-resistance exists among drugs within each class. ${ }^{3,4}$ In patients with resistant virus, use of antiretroviral agents from new classes offers considerable potential benefit because of the absence of cross-resistance. ${ }^{5-7}$

HIV-1 integrase represents a new therapeutic target. ${ }^{8,9}$ Consequently, HIV-1 integrase inhibitors would be expected to retain activity against HIV-1 that is resistant to other classes of antiretroviral drugs. Raltegravir (MK-0518; Isentress, Merck), an HIV integrase strand-transfer inhibitor, has exhibited substantial efficacy and a favorable safety profile in patients infected with HIV-1,7,10,11 including those with multidrugresistant HIV-1 and a history of treatment failure.,11 In a phase 2 study of previously treated patients who had multidrug-resistant virus, mean decreases in the viral load at week 24 ranged from 1.8 to $1.9 \log _{10}$ copies per milliliter in those receiving raltegravir at doses of 200, 400, or $600 \mathrm{mg}$ twice daily in combination with optimized background therapy, as compared with $0.4 \log _{10}$ copies per milliliter in those receiving optimized background therapy alone; in approximately $60 \%$ of patients receiving raltegravir, as compared with $13 \%$ of patients receiving placebo, HIV-1 RNA levels were reduced to less than 50 copies per milliliter. ${ }^{7}$ These findings were confirmed in both phase 3 BENCHMRK-1 and BENCHMRK-2 studies (Blocking Integrase in Treatment Experienced Patients with a Novel Compound against HIV, Merck studies). ${ }^{11}$ In this report, we present the results of subgroup efficacy analyses and the evaluation of virologic resistance to raltegravir in patients with virologic failure, using data from week 48 from the combined BENCHMRK-1 and BENCHMRK-2 studies.

\section{METHODS}

The studies were designed, managed, and analyzed by the sponsor in conjunction with the academic authors. The authors had access to all study data on request. This report was principally drafted by two academic authors and four industry authors and was critically reviewed and approved by all the authors in its final form before submission. All authors vouch for the completeness and accuracy of the data.

\section{STUDY DESIGN}

The identical study design of BENCHMRK-1 and BENCHMRK-2, criteria for scoring the activity of optimized background therapy, and the overall evaluation of efficacy are described in detail in the article by Steigbigel et al. in this issue of the Journal. ${ }^{11}$ The subgroup efficacy analyses included complete 48-week data from both studies. The potential emergence of resistance to raltegravir was investigated in patients with virologic failure by genotyping the integrase coding sequence. The integrase gene was reverse-transcribed from plasma HIV-1 RNA and sequenced according to standard methods. Consensus amino acid sequences were compared with pretreatment genotypes.

\section{STATISTICAL ANALYSIS}

Because BENCHMRK-1 and BENCHMRK-2 shared the same study design and showed consistent treatment effects, a combined analysis and exploratory subgroup analyses of pooled data from the two studies were undertaken. Subgroup analyses based on potential prognostic factors were prespecified (except for the combination of enfuvirtide and darunavir in optimized background therapy) before unblinding of the data. The phenotypic and genotypic sensitivity scores are the total number of antiretroviral drugs used as part of the optimized background therapy to which a patient's HIV was fully susceptible, as determined with the use of phenotypic and genotypic resistance testing, respectively. Enfuvirtide use in optimized background therapy in patients who had not previously received enfuvirtide was counted as one active drug in optimized background therapy and added to the genotypic and phenotypic sensitivity scores. Darunavir use in optimized background therapy in patients who had not previously received darunavir was likewise counted as one active drug and added to the phenotypic and genotypic sensitivity scores. In addition, darunavir use in optimized background therapy in patients who had not previously received darunavir was counted as one active protease inhibitor. 
Prognostic factors, such as baseline HIV-1 RNA levels and the genotypic and phenotypic sensitivity scores for optimized background therapy, may be strongly associated with virologic and immunologic responses but are less likely to be associated with adverse events or discontinuation for reasons not related to treatment. Because it predominantly reflects the antiretroviral effect of treatment, an observed-failure approach (in which only patients who discontinued the study because of lack of efficacy were considered to have treatment failure at subsequent time points) was used as the basis of the exploratory subgroup analyses. ${ }^{7}$ Differences (and the 95\% confidence intervals) between treatment groups in the proportion of patients with HIV-1 RNA levels below 50 copies per milliliter and in the mean change from the baseline CD4 cell counts were calculated for both the overall study groups and subgroups of interest. ${ }^{11}$

\section{RESULTS}

\section{CHARACTERISTICS OF THE PATIENTS}

A total of 699 patients were treated in BENCHMRK-1 and BENCHMRK-2: 462 patients received raltegravir and 237 received placebo. Baseline characteristics were generally balanced between the treatment groups within each study. ${ }^{11}$ Table 1 lists selected baseline characteristics that have been identified as factors potentially affecting the efficacy of antiretroviral therapy. These covariates include the baseline HIV-1 RNA level and CD4 cell count; the use or nonuse of newly available antiretroviral drugs such as enfuvirtide, darunavir, and tipranavir as part of optimized background therapy; the number of active protease inhibitors used in optimized background therapy; and the number of active drugs in optimized background therapy, as measured by the phenotypic or genotypic sensitivity score at baseline.

Baseline HIV-1 RNA levels were greater than 100,000 copies per milliliter in $35 \%$ of raltegravir recipients and $33 \%$ of placebo recipients. Baseline CD4 cell counts were 50 or less per cubic millimeter in $32 \%$ and $33 \%$ of raltegravir and placebo recipients, respectively. The median number of antiretroviral drugs in the optimized background therapy was four in both treatment groups. Despite this aggressive treatment, 36\% of patients in the raltegravir group and $41 \%$ of patients in the placebo group had no fully active protease inhibitors in their optimized background therapy. The genotypic sensitivity score of the optimized background therapy was 0 in $25 \%$ of patients receiving raltegravir and in $27 \%$ of patients receiving placebo. The phenotypic sensitivity score of the optimized background therapy was 0 in $15 \%$ of raltegravir recipients and in $19 \%$ of placebo recipients.

\section{SUBGROUP EFFICACY ANALYSES}

As reported in the article by Steigbigel et al., ${ }^{11}$ the overall virologic and immunologic responses in the raltegravir groups were superior to those in the placebo groups after 16 weeks and 48 weeks of treatment. At week 48, in the analysis in which noncompletion constituted treatment failure, $72 \%$ of patients in the raltegravir groups, as compared with $37 \%$ of patients in the placebo groups, had HIV-1 RNA levels below 400 copies per milliliter $(\mathrm{P}<0.001)$, and $62 \%$ of patients in the raltegravir groups, as compared with $33 \%$ of patients in the placebo groups, had HIV-1 RNA levels below 50 copies per milliliter $(\mathrm{P}<0.001)$. Similar results were seen in the analyses that used the observed-failure approach.

In subgroup analyses involving selected baseline prognostic factors and demographic characteristics based on the observed-failure approach, raltegravir recipients had higher virologic and immunologic response rates than placebo recipients across most subgroups, including patients with baseline HIV-1 RNA levels of more than 100,000 copies per milliliter and CD4 cell counts of less than 50 per cubic millimeter (Fig. 1). In patients receiving optimized background therapy with a genotypic sensitivity score of 0 , HIV-1 RNA levels of less than 50 copies per milliliter were achieved at week 48 in $45 \%$ of patients receiving raltegravir as compared with $3 \%$ of those receiving placebo, and the mean changes in the CD4 cell count between baseline and week 48 were 81 and 11 per cubic millimeter, respectively (Fig. 2). Among patients with more active optimized background therapy, reflected by a genotypic sensitivity score of $2,77 \%$ of raltegravir recipients had HIV-1 RNA levels below 50 copies per milliliter at week 48 , as compared with $62 \%$ of placebo recipients; the corresponding mean changes in the CD4 cell count were 145 and 87 per cubic millimeter, respectively. Similar findings were observed in the subgroup analysis based on the phenotypic sensitivity score. Only 
Table 1. Selected Baseline Characteristics of the Patients in BENCHMRK-1 and BENCHMRK-2.*

Raltegravir Groups

$(\mathrm{N}=462)$

Placebo Groups

Characteristic

no. (\%)

Sex

Male

405 (88)

57 (12)

$210(89)$

Female

Race or ethnic group ${ }^{-}$

White

$300(65)$

$173(73)$

Black

66 (14)

$26(11)$

Asian

$16(3)$

6 (3)

Hispanic

53 (11)

$19(8)$

Other

27 (6)

$13(5)$

Region

North America

192 (42)

99 (42)

South America

61 (13)

31 (13)

Asia or Australia

$38(8)$

$20(8)$

Europe

$171(37)$

87 (37)

Viral subtype

Clade B

$415(90)$

219 (92)

Other

$15(6)$

Missing data

$8(2)$

$3(1)$

Plasma HIV-1 RNA level

$\leq 50,000$ copies $/ \mathrm{ml}$

217 (47)

125 (53)

$>50,000$ and $\leq 100,000$ copies $/ \mathrm{ml}$

$81(18)$

34 (14)

$>100,000$ copies $/ \mathrm{ml}$

$164(35)$

$78(33)$

CD4 cell count

$\leq 50 / \mathrm{mm}^{3}$

$146(32)$

$78(33)$

$>50$ and $\leq 200 / \mathrm{mm}^{3}$

173 (37)

$85(36)$

$>200 / \mathrm{mm}^{3}$

$142(31)$

$74(31)$

Missing data

$1(<1)$

0

Enfuvirtide use in OBT

No

Yes, in those who had previously used enfuvirtide

Yes, in those who had not used enfuvirtide

$92(20)$

$48(20)$

Darunavir use in OBT

\section{No}

$278(60)$

$138(58)$

Yes, in those who had previously used darunavir

$18(4)$

$9(4)$

Yes, in those who had not used darunavir

$166(36)$

$90(38)$

Tipranavir use in OBT

No

364 (79)

$193(81)$

Yes

By phenotypic test

HIV-1 resistant to tipranavir

$41(9)$

$19(8)$

HIV-l sensitive to tipranavir

$53(11)$

25 (11)

Missing data

$4(1)$

0

By genotypic test

HIV-I resistant to tipranavir

$44(10)$

22 (9)

HIV-I sensitive to tipranavir

$53(11)$

$22(9)$

Missing data

$1(<1)$

0

The New England Journal of Medicine 


\begin{tabular}{|c|c|c|}
\hline \multicolumn{3}{|c|}{ Table 1. (Continued.) } \\
\hline \multirow[t]{2}{*}{ Characteristic } & $\begin{array}{l}\text { Raltegravir Groups } \\
\qquad(\mathrm{N}=462)\end{array}$ & $\begin{array}{l}\text { Placebo Groups } \\
(\mathrm{N}=\mathbf{2 3 7})\end{array}$ \\
\hline & \multicolumn{2}{|c|}{ no. (\%) } \\
\hline \multicolumn{3}{|c|}{ No. of active protease inhibitors in OBT (by phenotypic test) $\ddagger$} \\
\hline 0 & $168(36)$ & $98(41)$ \\
\hline$\geq 1$ & $278(60)$ & $137(58)$ \\
\hline Missing data & $16(3)$ & $2(1)$ \\
\hline \multicolumn{3}{|c|}{ Phenotypic sensitivity score $\mathbb{Z}$} \\
\hline 0 & $69(15)$ & $44(19)$ \\
\hline 1 & $145(31)$ & $72(30)$ \\
\hline 2 & $142(31)$ & $66(28)$ \\
\hline$\geq 3$ & $85(18)$ & $48(20)$ \\
\hline Missing data & $21(5)$ & $7(3)$ \\
\hline \multicolumn{3}{|c|}{ Genotypic sensitivity score $\llbracket$} \\
\hline 0 & $115(25)$ & $66(28)$ \\
\hline 1 & 178 (39) & $96(41)$ \\
\hline 2 & $111(24)$ & $49(21)$ \\
\hline$\geq 3$ & $51(11)$ & $23(10)$ \\
\hline Missing data & $7(2)$ & $3(1)$ \\
\hline \multicolumn{3}{|c|}{$\begin{array}{l}\text { OBT denotes optimized background therapy. } \\
\text { Race or ethnic group was self-reported. } \\
\text { Darunavir in OBT in patients who had not previously received darunavir was counted as one active protease inhibitor. } \\
\text { First use of enfuvirtide in OBT was counted as one active drug and added to the phenotypic and genotypic sensitivity } \\
\text { scores. First use of darunavir in OBT was counted as one active drug and added to the phenotypic and genotypic sensi- } \\
\text { tivity scores. }\end{array}$} \\
\hline
\end{tabular}

in the relatively few patients whose optimized background therapy was associated with a genotypic or phenotypic sensitivity score of 3 or more was the treatment advantage of raltegravir over placebo not strikingly evident.

Additional analyses were conducted to evaluate the effect of newly available antiretroviral drugs (darunavir or enfuvirtide used alone or in combination, and tipranavir) as part of optimized background therapy. Among patients receiving both enfuvirtide and darunavir for the first time, $89 \%$ of patients in the raltegravir groups and $68 \%$ of patients in the placebo groups had HIV-1 RNA levels of less than 50 copies per milliliter at week 48 (Fig. 3), with corresponding changes in the CD4 cell count of 129 and 81 per cubic millimeter, respectively (Fig. 1 in Supplementary Appendix 2, available with the full text of this article at www.nejm. org). HIV-1 RNA levels below 50 copies per milliliter were achieved in $69 \%$ and $80 \%$ of the raltegravir recipients as compared with $47 \%$ and $57 \%$ of the placebo recipients using either darunavir or enfuvirtide for the first time, respective- ly, and the changes in CD4 cell count were 114 and 116 per cubic millimeter among raltegravir recipients as compared with 74 and 49 per cubic millimeter among placebo recipients, respectively.

Raltegravir recipients had higher response rates than placebo recipients, whether or not tipranavir was used in the optimized background therapy. ${ }^{12}$ In patients treated with tipranavir who had virus that was genotypically sensitive to tipranavir, HIV-1 RNA levels were reduced to less than 50 copies per milliliter in $73 \%$ of raltegravir recipients, as compared with $40 \%$ of placebo recipients (Fig. 3). The mean changes in the CD4 cell count in this subgroup were 114 and 56 per cubic millimeter, respectively (Fig. 1 in Supplementary Appendix 2). In patients treated with tipranavir as part of their optimized background therapy who had virus that was genotypically resistant to tipranavir, $36 \%$ of raltegravir recipients, as compared with $15 \%$ of placebo recipients, had HIV-1 RNA levels of less than 50 copies per milliliter; the corresponding mean changes in the CD4 count were 79 and 12 per cubic millimeter, respectively. 


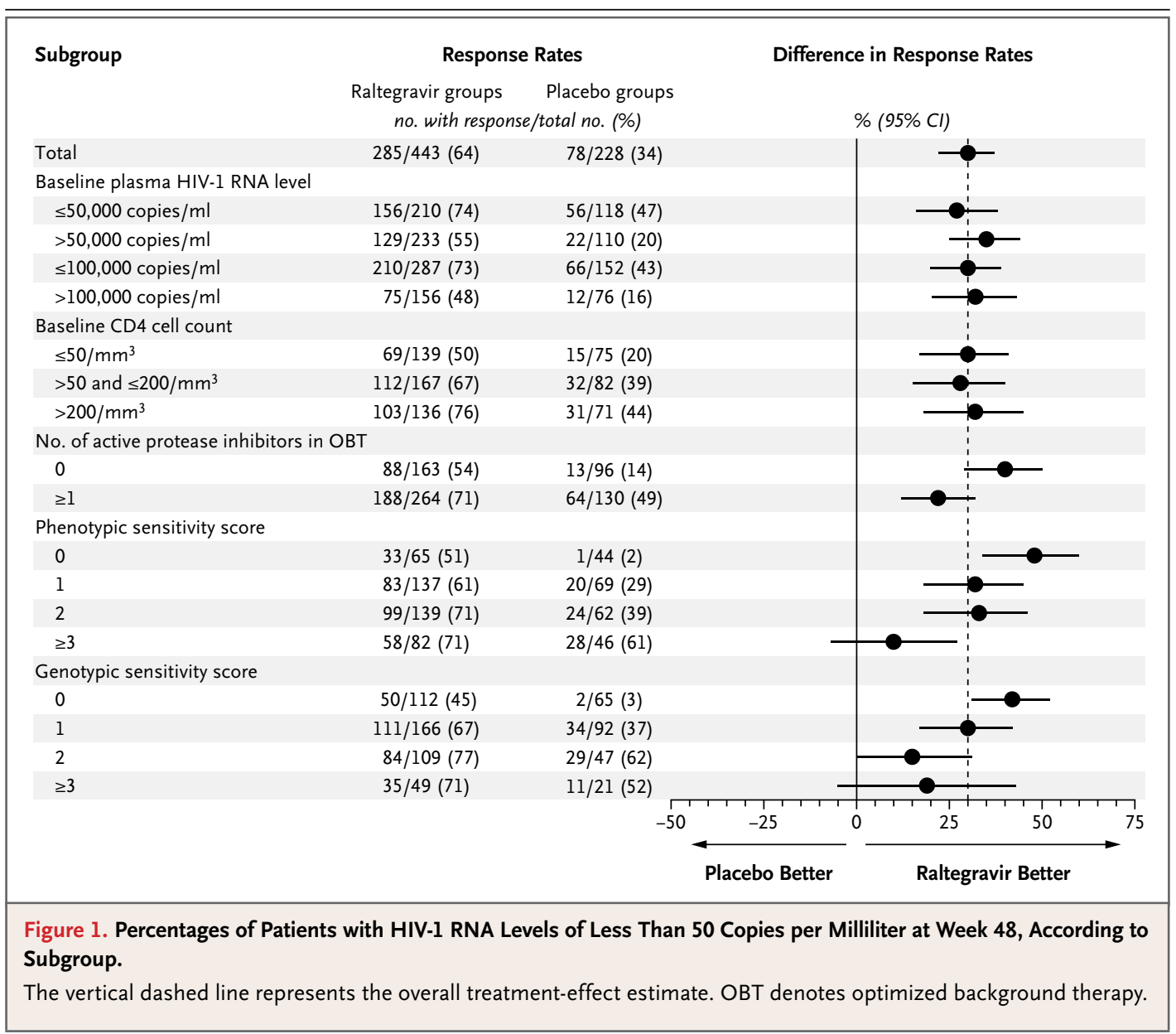

Additional efficacy analyses based on sex, race or ethnic group, geographic region, and viral subtype consistently showed higher response rates in the raltegravir groups than in the placebo groups, in terms of viral-load reductions (Fig. 4) as well as increases in the CD4 cell count (Fig. 2 in Supplementary Appendix 2).

\section{RESISTANCE MUTATIONS IN THE INTEGRASE GENE}

A total of 105 of the 462 patients receiving raltegravir $(23 \%)$ had virologic failure by week 48 . Integrase genotyping had been performed both at baseline and after virologic failure in 94 of these 105 patients $(90 \%)$ at the time of this analysis; 64 of the 94 patients $(68 \%)$ had genotypic evidence of viral resistance to raltegravir when tested at the time of, or shortly after, viral rebound (Table 2). Of the 64 patients with integrase mutations previously associated with phenotypic raltegravir resistance, 48 (75\%) had two or more such mutations. Virologic failure was generally associated with mutations at one of three residues - Y143, Q148, or N155 - usually in combination with at least one other mutation (Table 2). The risk of mutations arising during treatment was increased in patients with higher baseline HIV-1 RNA levels or receiving optimized background therapy with a genotypic or phenotypic sensitivity score of 0 .

\section{I SCUSSION}

The results of the BENCHMRK- 1 and BENCHMRK-2 studies showed the consistently superior efficacy of raltegravir over placebo when used in combination with optimized background therapy. ${ }^{11}$ Substantial viral-load suppression and increases in CD4 cell counts as compared with the baseline values were sustained for at least 48 weeks. The absolute response rate in the combined placebo groups (33\% of patients having $<50$ HIV-1 RNA copies per milliliter) was better 


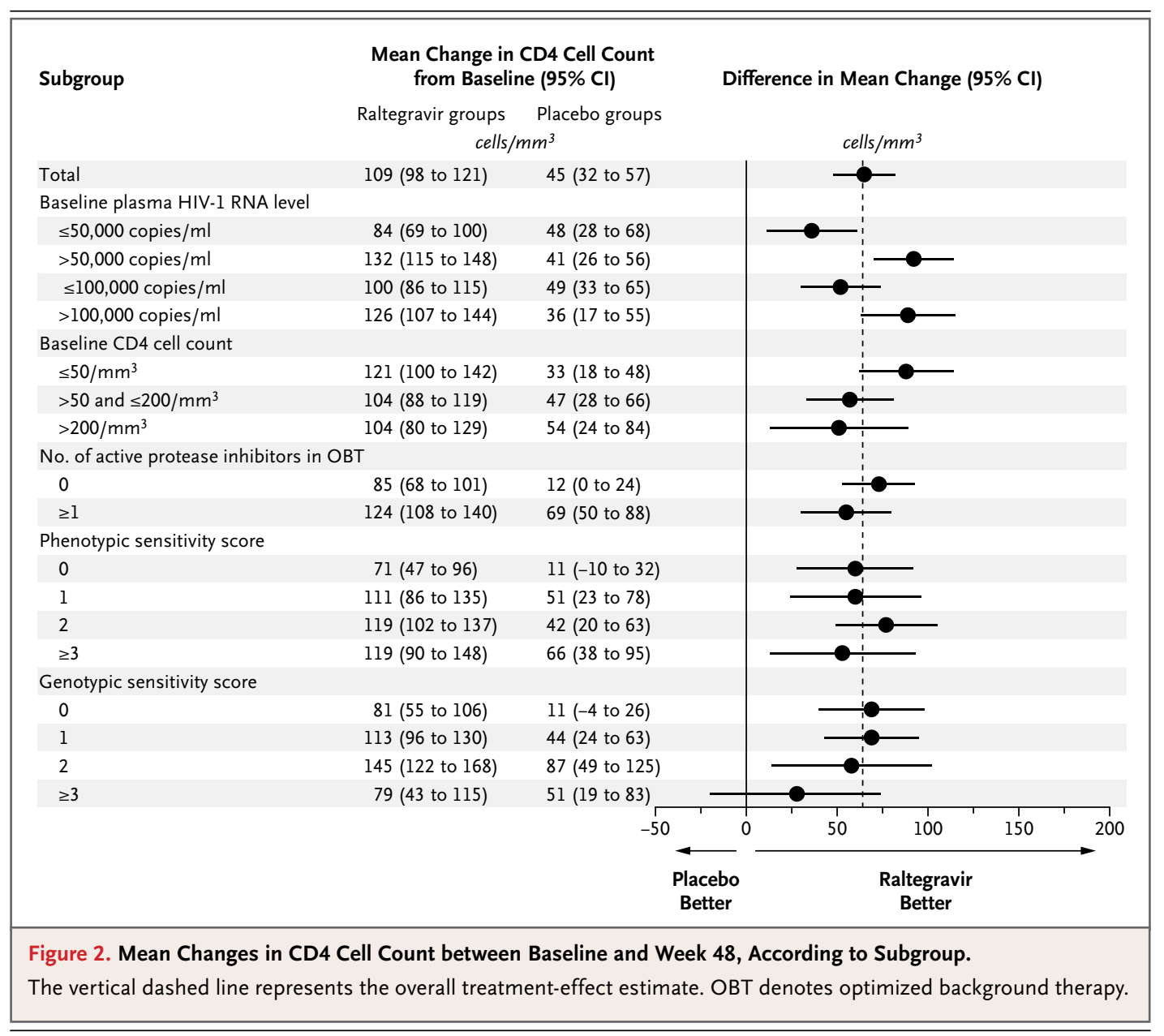

than that in a phase 2 study of raltegravir (13\% with $<50$ HIV-1 RNA copies per milliliter), ${ }^{7}$ most likely reflecting the permitted use of investigational or recently licensed antiretroviral drugs in optimized background therapy in the BENCHMRK studies. The response rate in the placebo groups in these studies was consistent with the $45 \%$ response rate reported previously among similar patients receiving darunavir. ${ }^{13}$

The BENCHMRK studies were not powered to show significant effects within subgroups. ${ }^{14} \mathrm{Al}-$ though there may have been imbalances within each subgroup in other prognostic factors between the raltegravir groups and the placebo groups, the subgroup analyses consistently showed a treatment advantage of raltegravir over placebo when given in combination with optimized background therapy. Specifically, raltegravir was more efficacious than placebo, regardless of baseline prognostic factors. Overall, patients with lower baseline HIV-1 RNA levels or higher baseline CD4 cell counts had higher response rates than patients with higher baseline HIV-1 RNA levels or lower baseline CD4 cell counts; however, the differences in treatment effect between the raltegravir and placebo groups were consistent in direction and magnitude in these subgroups. These findings are similar to those from the T-20 vs. Optimized Regimen Only Studies (TORO-1 and TORO-2) of enfuvirtide. ${ }^{5,6,15}$

As found in the phase 2 trial of raltegravir in previously treated patients infected with a multidrug-resistant virus, ${ }^{7}$ raltegravir had superior efficacy as compared with placebo in patients receiving optimized background therapy with a genotypic or phenotypic sensitivity score of 0 , which is generally regarded as the most challenging treatment scenario. Nonetheless, functional monotherapy with raltegravir (in which there are no fully active drugs in the optimized back- 


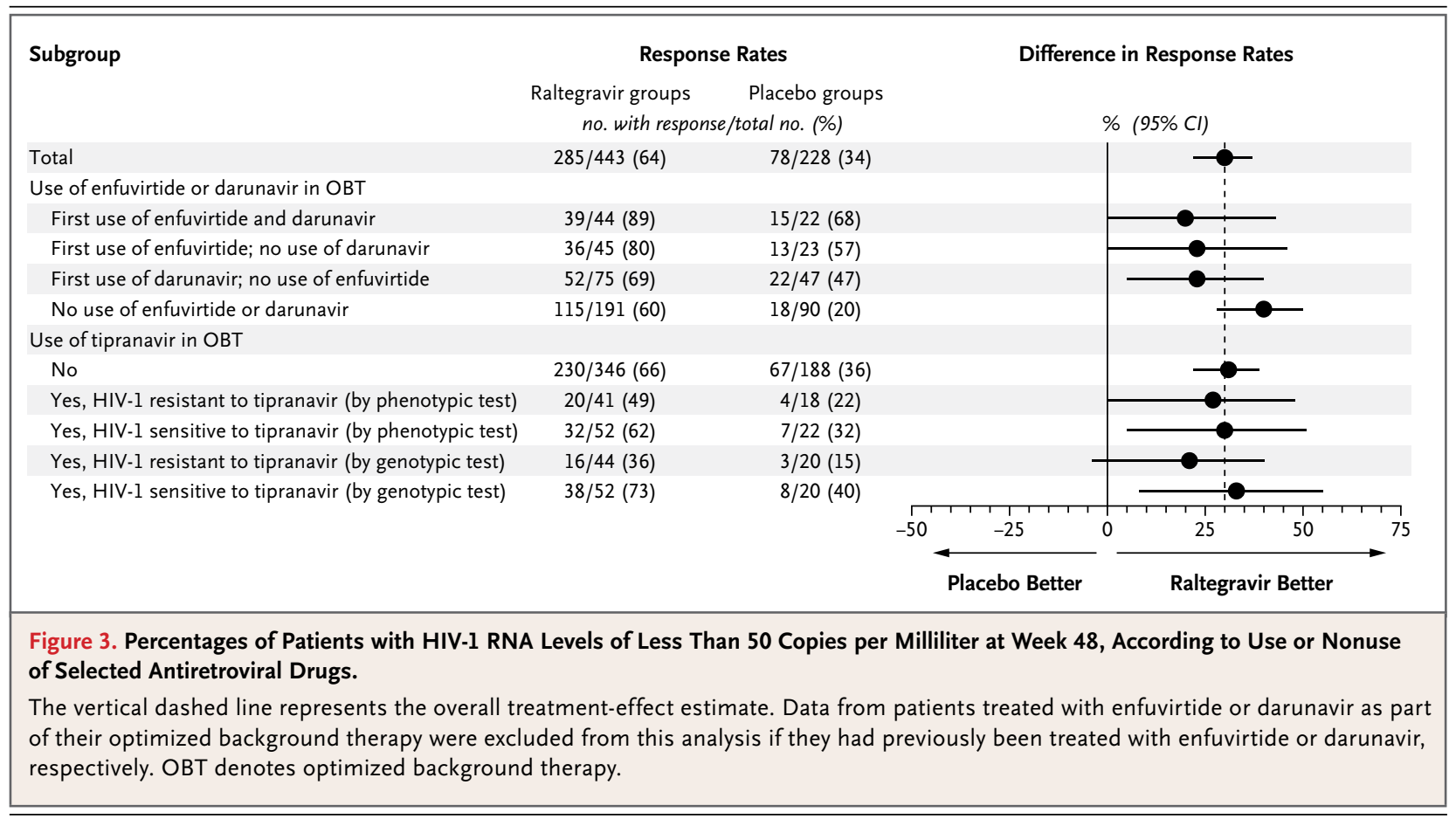

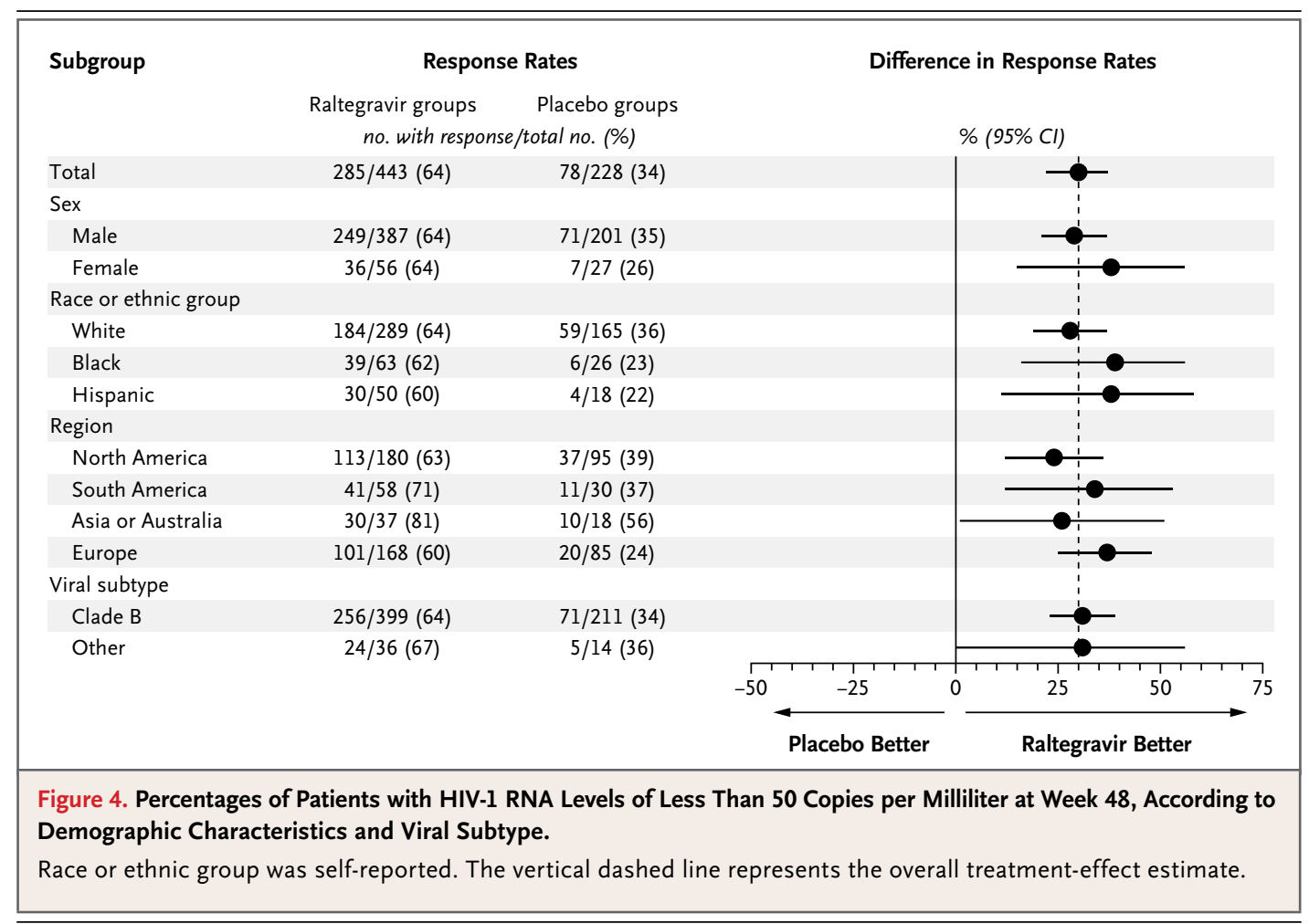

ground therapy) should be avoided whenever phenotypic sensitivity score $>0$ ) as well as the possible, given the greater absolute response lower risk of virologic failure and development rates among patients with more active optimized of resistance in patients receiving more active background therapy (defined as genotypic or optimized background therapy. For example, in 


\begin{tabular}{|c|c|c|c|c|c|c|}
\hline \multirow[t]{3}{*}{ Resistance Mutation in HIV Integrase Gene } & $\begin{array}{l}\text { Integrase } \\
\text { Genotyping } \\
\text { Performed } \\
(\mathrm{N}=94)\end{array}$ & \multicolumn{3}{|c|}{ Genotypic Sensitivity Score at Baseline } & \multirow[t]{3}{*}{$\begin{array}{l}\text { Nonresponse } \\
(N=13) \dagger\end{array}$} & \multirow[t]{3}{*}{$\begin{array}{l}\text { Viral Rebound } \\
\qquad(\mathrm{N}=81) \div\end{array}$} \\
\hline & & $0(\mathrm{~N}=49)$ & $1(\mathrm{~N}=27)$ & $\geq 2(N=18)$ & & \\
\hline & \multicolumn{4}{|c|}{ number of patients (percent) } & & \\
\hline Mutation known to confer raltegravir resistance $\mathbb{}$ & $64(68)$ & $38(78)$ & $20(74)$ & $6(33)$ & & \\
\hline No amino acid changes from baseline sequence & $25(27)$ & $9(18)$ & $6(22)$ & $10(56)$ & & \\
\hline $\begin{array}{c}\text { Amino acid changes of unknown phenotypic } \\
\text { effect }\end{array}$ & $5(5)$ & $2(4)$ & $1(4)$ & $2(11)$ & & \\
\hline \multicolumn{7}{|l|}{ Specific mutation } \\
\hline At amino acid 148,155 , or 143 & & & & & $8(62)$ & $54(67)$ \\
\hline Amino acid 148 & & & & & $3(23)$ & $24(30)$ \\
\hline Q148H & & & & & $1(8)$ & $12(15)$ \\
\hline Q148K & & & & & $1(8)$ & $4(5)$ \\
\hline Q148R & & & & & $1(8)$ & $14(17)$ \\
\hline Amino acid 155 & & & & & $3(23)$ & $35(43)$ \\
\hline Amino acid 143 & & & & & $2(15)$ & $8(10)$ \\
\hline No mutation at amino acid 148,155 , or 143 & & & & & $5(38)$ & $27(33)$ \\
\hline Other raltegravir-resistance mutation $\|$ & & & & & 0 & $2(2)$ \\
\hline $\begin{array}{l}\text { Amino acid changes of unknown phe- } \\
\text { notypic effect }\end{array}$ & & & & & $1(8)$ & $4(5)$ \\
\hline $\begin{array}{l}\text { No amino acid changes from baseline } \\
\text { sequence }\end{array}$ & & & & & $4(31)$ & $21(26)$ \\
\hline
\end{tabular}

* Of the 462 raltegravir recipients, 105 (23\%) had virologic failure by week 48; of these, 94 (89\%) had genotyping results available at both baseline and the time of virologic failure and were included in this analysis. Four patients for whom only baseline genotypes were available were not included.

$\uparrow$ A lack of response was defined as an HIV RNA $\log _{10}$ level that was not reduced by more than 1.0 , or that was not suppressed to less than 400 copies per milliliter, by week 16 .

$\$$ Viral rebound was defined at or after week 16 as an HIV-1 RNA level of more than 400 copies per milliliter (in two consecutive measurements at least 1 week apart) after an initial reduction to less than 400 copies per milliliter, or an increase of more than $1.0 \log _{10}$ HIV RNA level above the nadir level (on two consecutive measurements at least 1 week apart).

$\int$ Integrase mutations were $\mathrm{N} 155 \mathrm{H}, \mathrm{Q} 148 \mathrm{H}, \mathrm{Q} 148 \mathrm{~K}, \mathrm{Q} 148 \mathrm{R}, \mathrm{Y} 143 \mathrm{C}, \mathrm{Y} 143 \mathrm{R}$, or E92Q, usually in combination with other mutations.

9 Four patients had a single amino acid change (I203M in three and G163R in one), and one other patient had multiple changes (S24N, M50I, G70D, and L234I); these mutations have not yet been tested in phenotypic assays.

|| One patient had the E92E/Q mixture, and one had the L74M and E92Q mutations.

$89 \%$ of patients receiving raltegravir along with enfuvirtide and darunavir for the first time in their optimized background therapy, HIV-1 RNA levels were reduced to less than 50 copies per milliliter. This substantial rate of viral suppression, which approaches the rates reported among patients infected with HIV who have not been previously treated, ${ }^{16}$ is remarkable for patients infected with virus that has triple-class drug resistance and in whom therapy has failed previously. The BENCHMRK studies, along with other recently reported studies, ${ }^{13,17}$ indicate that suppressing the viral load to less than 50 copies per milliliter is an attainable goal, even in heavily pretreated patients infected with virus that has multiclass drug resistance.

In healthy volunteers, coadministration of raltegravir with tipranavir reduced nadir levels of raltegravir by $55 \% .{ }^{12}$ Therefore, a detailed analysis of tipranavir use in the phase $3 \mathrm{BENCH}-$ MRK studies was undertaken to evaluate the potential effect on the efficacy of raltegravir. Among patients who received tipranavir as part of optimized background therapy in both the raltegravir and the placebo groups, those with tipranavir-resistant virus had lower response 
rates than those with tipranavir-sensitive virus, presumably owing to the lack of an active protease inhibitor in the tipranavir-based optimized background therapy. In patients with tipranavirsensitive virus, the use of tipranavir as part of optimized background therapy did not appear to negate the favorable treatment effect in the raltegravir groups.

Sex, race or ethnic group, geographic region, and viral subtype (clade B vs. other clades) apparently did not affect overall efficacy. Subgroup analyses according to sex, race or ethnic group, geographic region, and viral subtype showed generally higher response rates for raltegravir recipients than for placebo recipients, but more research is necessary to confirm these observations.

The limited genotypic-resistance data available to date support the in vitro findings that more than one integrase mutation is generally observed in HIV strains that become resistant to raltegravir. ${ }^{9}$ Genotypic resistance to raltegravir was anticipated in patients who had virologic failure in the raltegravir groups, especially in those who received raltegravir as functional monotherapy. Consequently, as recommended in formal treatment guidelines for all antiretroviral drugs, ${ }^{1,2}$ raltegravir should be used in combination with at least one other active antiretroviral agent whenever possible to minimize the development of resistance and the risk of virologic failure. Long-term efficacy data, beyond 48 weeks, particularly in patients receiving optimized background therapy with genotypic and phenotypic sensitivity scores of 0 , will aid in the interpretation of specific integrase mutations and their contribution to the development of resistance to raltegravir.

In summary, treatment with raltegravir in combination with optimized background therapy, as compared with optimized background therapy alone, resulted in significant virologic and immunologic benefits for at least 48 weeks in both international BENCHMRK studies. The subgroup analyses presented here show a consistently favorable treatment effect of raltegravir, regardless of viral load, CD4 cell count, and genotypic or phenotypic sensitivity score at baseline and regardless of whether enfuvirtide, darunavir, or both were included in the optimized background therapy. Our data provide evidence that raltegravir will be a valuable addition to the current armamentarium for the treatment of patients infected with multidrug-resistant HIV-1.

\section{Supported by Merck.}

Dr. Cooper reports serving as an investigator for and receiving speaker's and advisory fees from Merck; Dr. Steigbigel, serving as an investigator for Merck, Pfizer, and Boehringer Ingelheim; Dr. Gatell, receiving grant support or lecture or advisory fees from Merck, Roche, GlaxoSmithKline, Bristol-Myers Squibb, Tibotec, Pfizer, Gilead, Abbott, and Boehringer Ingelheim; Dr. Rockstroh, receiving lecture or advisory fees from Merck, Roche, GlaxoSmithKline, Bristol-Myers Squibb, Tibotec, Pfizer, Gilead, Abbott, and Boehringer Ingelheim; Dr. Katlama, receiving advisory or lecture fees from Merck, Gilead, Roche, GlaxoSmithKline, Tibotec, Bristol-Myers Squibb, and Boehringer Ingelheim; Dr. Yeni, receiving grant support and advisory fees from Tibotec, Merck, GlaxoSmithKline, Pfizer, Gilead, Abbott, and Boehringer Ingelheim; Dr. Lazzarin, receiving advisory fees from Merck and consulting and lecture fees from Gilead, Roche, Bristol-Myers Squibb, GlaxoSmithKline, Tibotec, Boehringer Ingelheim, Pfizer, Merck, and Abbott; Dr. Clotet, receiving consulting and lecture fees from Gilead, Roche, Bristol-Myers Squibb, GlaxoSmithKline, Tibotec, Boehringer Ingelheim, Pfizer, Merck, and Abbott; Dr. Kumar, serving as an investigator for and receiving consulting and lecture fees from Merck; Dr. Eron, serving as an investigator for Merck, GlaxoSmithKline, Abbott, and Panacos and receiving consulting or lecture fees from Merck, Gilead, Bristol-Myers Squibb, GlaxoSmithKline, Abbott, Trimeris, Roche, Virco, Monogram, and Panacos; Dr. Schechter, serving as an investigator for Merck and receiving consulting fees or lecture fees from Merck, Gilead, Bristol-Myers Squibb, Abbott, Roche, and Pfizer; Dr. Markowitz, serving as an investigator for Merck, GlaxoSmithKline, Gilead, and Tibotec and receiving consulting fees from Merck, Gilead, GlaxoSmithKline, Sequoia Pharmaceuticals, and Quintiles and grant support from Merck and Pfizer; Dr. Loutfy, serving as an investigator for Merck; Dr. Lennox, serving as an investigator for Merck, Gilead, Pfizer, Tibotec, Schering, and Abbott and receiving consulting fees from Merck, Roche, and Abbott; and Drs. Zhao, Chen, Meibohm, Miller, Hazuda, DiNubile, Isaacs, Teppler, and Nguyen, Mr. Ryan, Mr. Rhodes, Mr. Killar, Ms. Gilde, Ms. Strohmaier, and Mr. Nessly, being employees of Merck and owning stock or stock options or both in the company. No other potential conflict of interest relevant to this article was reported.

We thank all the patients and their caregivers who participated in the BENCHMRK studies; the investigators who enrolled their patients in these trials for their important contributions; Keith Gottesdiener for his thoughtful advice; and Robert Danovich, Joann DiLullo, Karyn Davis, Anita Jain, Andrea Karian, Hong Wan, Jennifer Reinert, Bernard Akyena, Xia Xu, and Anthony Rodgers for their expert assistance.
REFERENCES

1. Panel on Antiretroviral Guidelines for Adults and Adolescents. Guidelines for the use of antiretroviral agents in HIV-1infected adults and adolescents. Washington, DC: Department of Health and Hu- man Services, January 29, 2008. (Accessed June 30, 2008, at http://aidsinfo.nih.gov/ contentfiles/AdultandAdolescentGL.pdf.) 2. Hammer SM, Saag MS, Schechter M, et al. Treatment for adult HIV infection:
2006 recommendations of the International AIDS Society-USA Panel. JAMA 2006; 296:827-43.

3. Tozzi V, Zaccarelli M, Bonfigli S, et al. Drug-class-wide resistance to antiretro- 
virals in HIV-infected patients failing therapy: prevalence, risk factors and virological outcome. Antivir Ther 2006;11:55360 .

4. Richman DD, Morton S, Wrin T, et al. The prevalence of antiretroviral drug resistance in the United States. AIDS 2004; 18:1393-401.

5. Lalezari JP, Henry K, O’Hearn M, et al. Enfuvirtide, an HIV-1 fusion inhibitor, for drug-resistant HIV infection in North and South America. N Engl J Med 2003;348: 2175-85. [Erratum, N Engl J Med 2003; 349:1100.]

6. Lazzarin A, Clotet B, Cooper D, et al. Efficacy of enfuvirtide in patients infected with drug-resistant HIV-1 in Europe and Australia. N Engl J Med 2003;348:218695.

7. Grinsztejn B, Nguyen BY, Katlama C, et al. Safety and efficacy of the HIV-1 integrase inhibitor raltegravir (MK-0518) in treatment-experienced patients with multidrug-resistant virus: a phase II randomised controlled trial. Lancet 2007;369:1261-9.

8. Hazuda DJ, Felock P, Witmer M, et al. Inhibitors of strand transfer that prevent integration and inhibit HIV-1 replication in cells. Science 2000;287:646-50.

9. Espeseth AS, Felock P, Wolfe A, et al. HIV-1 integrase inhibitors that compete with the target DNA substrate define a unique strand transfer conformation for integrase. Proc Natl Acad Sci U S A 2000; 97:11244-9.

10. Markowitz M, Nguyen B-Y, Gotuzzo E, et al. Rapid and durable antiretroviral effect of the HIV-1 integrase inhibitor raltegravir as part of combination therapy in treatment-naïve patients with HIV-1 infection: results of a 48-week controlled study. J Acquir Immune Defic Syndr 2007;46:12533.

11. Steigbigel RT, Cooper DA, Kumar PN, et al. Raltegravir with optimized background therapy for resistant HIV-1 infection. N Engl J Med 2008;359:339-54.

12. Food and Drug Administration. Antiviral Drugs Advisory Committee Meeting 9/5/2007: Merck, raltegravir tablets NDA 22-145 [applicant presentation]. (Accessed June 30, 2008, at http://www.fda.gov/ohrms/ dockets/ac/07/slides/2007-4314s1-04-merckisaacs.pdf.)
13. Clotet B, Bellos N, Molina J-M, et al. Efficacy and safety of darunavir-ritonavir at week 48 in treatment-experienced patients with HIV-1 infection in POWER 1 and 2: a pooled subgroup analysis of data from two randomised trials. Lancet 2007; 369:1169-78. [Erratum, Lancet 2008;371: 116.]

14. Wang R, Lagakos SW, Ware JH, Hunter DJ, Drazen JM. Statistics in medicine - reporting of subgroup analyses in clinical trials. N Engl J Med 2007;357:218994.

15. Montaner J, Guimaraes D, Chung J, Gafoor Z, Salgo M, DeMasi R. Prognostic staging of extensively pretreated patients with advanced HIV-1 disease. HIV Clin Trials 2005;6:281-90.

16. Gallant JE, DeJesus E, Arribas JR, et al. Tenofovir DF, emtricitabine, and efavirenz vs. zidovudine, lamivudine, and efavirenz for HIV. N Engl J Med 2006; 354:251-60.

17. MacArthur RD, Novak RM. Maraviroc: the first of a new class of antiretroviral agents. Clin Infect Dis 2008;47:236-41. Copyright (c) 2008 Massachusetts Medical Society.

FULL TEXT OF ALL JOURNAL ARTICLES ON THE WORLD WIDE WEB

Access to the complete text of the Journal on the Internet is free to all subscribers. To use this Web site, subscribers should go to the Journal's home page (www.nejm.org) and register by entering their names and subscriber numbers as they appear on their mailing labels. After this one-time registration, subscribers can use their passwords to log on for electronic access to the entire Journal from any computer that is connected to the Internet. Features include a library of all issues since January 1993 and abstracts since January 1975, a full-text search capacity, and a personal archive for saving articles and search results of interest. All articles can be printed in a format that is virtually identical to that of the typeset pages. Beginning 6 months after publication, the full text of all Original Articles and Special Articles is available free to nonsubscribers.

The New England Journal of Medicine 